\title{
ReaR
}

\section{Presión cricoidea o Sellick, ¿pasado o futuro de una maniobra cuestionada poco evaluada?}

Artículo original: Birenbaum A, Hajage D, Roche S, Ntouba A, Eurin M, Cuvillon P, et al. Effect of Cricoid Pressure Compared with a Sham Procedure in the Rapid Sequence Induction of Anesthesia: The IRIS Randomized Clinical Trial. JAMA surgery. 2018; PMID:30347104 (PubMed)

González Echevarría C, Rodríguez Durá P, Merino Martín I, Domínguez Bronchal MJ.

Complejo Hospitalario Universitario de Toledo

\section{Resumen}

La presión cricoidea es una maniobra ampliamente recomendada y extendida a la hora de ciertas intubaciones orotraqueales. Pero son pocos los estudios que hayan comparado su realización frente a no realizarla. Por tanto, aquellos profesionales dedicados a la vía aérea deben comprobar su eficacia. El estudio aquí analizado no consigue demostrar la no inferioridad de la intubación sin presión cricoidea durante la inducción de secuencia rápida (ISR). De modo que se alcanza un nuevo reto en el futuro: dejar de hacer lo que hemos aprendido haciendo. Por lo que probablemente serán las generaciones de residentes actuales los que dejarán como anecdótico una práctica cuestionada pero muy realizada.

\section{Introducción}

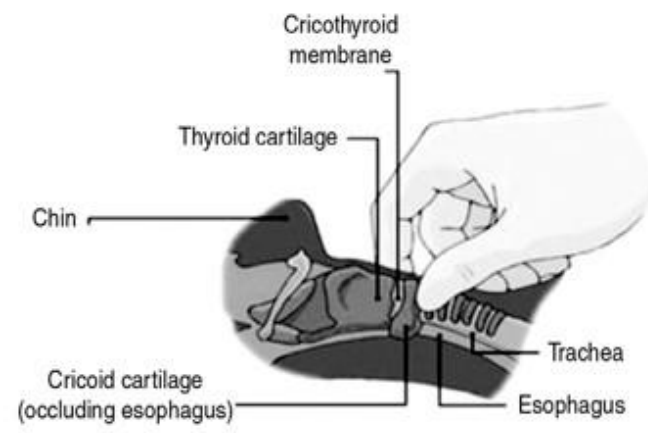

La presión cricoidea es una maniobra ampliamente recomendada y extendida a la hora de ciertas intubaciones orotraqueales. Pero son pocos los estudios que hayan comparado su realización frente a no realizarla. Por tanto, aquellos profesionales dedicados a la vía aérea deben comprobar su eficacia. El estudio aquí analizado no consigue demostrar la no inferioridad de la intubación sin presión cricoidea durante la inducción de secuencia rápida (ISR). De modo que se alcanza un nuevo reto en el futuro: dejar de hacer lo que hemos aprendido haciendo. Por lo que probablemente serán las generaciones de residentes actuales los que dejarán como anecdótico una práctica cuestionada pero muy realizada.

La maniobra de Sellick (o presión cricoidea) fue descrita por el anestesiólogo Brian Arthur Sellick (1961) basando sus estudios en un proceso exclusivamente fisiológico. Él mismo, la describió como: "la realización de presión sobre el cartílago cricoides para ocluir el esófago superior contra las vértebras cervicales $\left(5^{a}\right)$ y así prevenir la regurgitación de contenido gástrico a la faringe" (1). 


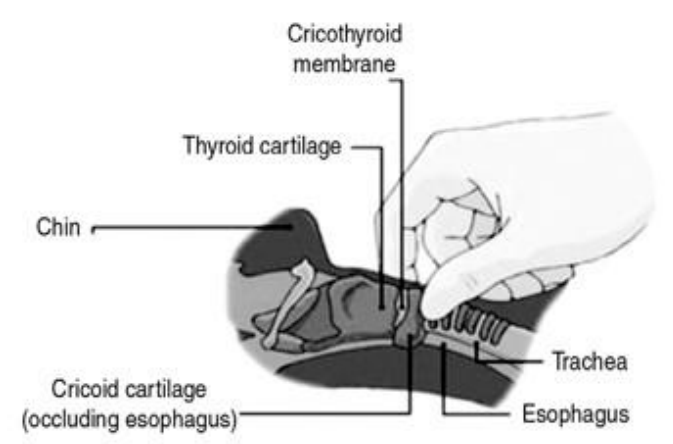

Figura 1: Huh, Y, Cook-jong Lee, J. Maniobra de Sellick. Aplicación de presión sobre cartílago cricoides [Internet]. 2015 [citado 20 enero 2020]. Disponible en: https://www.researchgate.net/figure/Sellickmaneuver-The-application-of-pressure-to-thecricoid-cartilage_fig3_309818535 (2)

A pesar de tratarse de una técnica descrita hace casi 60 años, y ampliamente generalizada, ningún estudio había evaluado seriamente dicha técnica. De manera que durante todos estos años se ha empleado dicho método en base a una creencia de utilidad y se ha considerado como un estándar de seguridad del paciente. Pero ¿qué ocurre si no se realiza la maniobra de Sellick? ¿Existe un aumento de complicaciones en su ausencia? ¿El Sellick realmente dificulta la intubación? ¿Alarga el tiempo de intubación la maniobra de presión cricoidea?

Hoy en día existe una amplia discusión, porque su eficacia está pobremente documentada y en múltiples estudios se ha evidenciado que su realización no disminuye la aspiración bronquial, dificulta la visión laringoscópica e incluso puede llegar a obstruir la vía aérea. Además, bajo realización de Sellick se ha observado riesgo aumentado de aspiración por oclusión parcial esofágica y aumento de presión en el esfínter esofágico inferior, e incluso, lesiones a nivel esofágico $\mathrm{y} / \mathrm{o}$ cricoideo (3).

Por tanto, este estudio es importante por los retos científicos a los que deberíamos haber hecho frente hace tiempo. Aunque los autores tuvieron que alcanzar un objetivo bastante difícil: lograr una gran muestra debido a la baja incidencia de aspiración bronquial bajo anestesia general $\mathrm{y}$ comparar la realización de presión cricoidea frente a no realizarla.

\section{Método}

Se trata de un ensayo clínico de noinferioridad,

multicéntrico, aleatorizado y doble ciego desarrollado en Francia. El reclutamiento comenzó en febrero de 2014 hasta febrero de 2017, recogiéndose el consentimiento informado bajo las normas éticas. Por tanto, la población diana fue cualquier paciente que requiriese una operación bajo anestesia general con inducción de secuencia rápida.

- Criterios de inclusión: mayor de 18 años con estómago lleno (menos de 6 horas de ayuno - no realizan diferencia entre líquidos y sólidos) o al menos un factor de riesgo para aspiración pulmonar (situación emergente, Índice de Masa Corporal $\quad(\mathrm{IMC})>30$, cirugía gástrica previa, íleo, puerperio precoz $\quad(<48$ horas $)$, gastroparesia diabética, reflujo gastroesofágico, hernia de hiato, náuseas $y$ vómitos preoperatorios y dolor).

- Criterios de exclusión: decisión del paciente, menores de 18 años, embarazadas, participación en otro ensayo clínico aleatorizado, ausencia de tarjeta del seguro nacional salud, contraindicación de uso succinilcolina o realización de presión cricoidea, neumonía o contusión pulmonar, anormalidad de la vía aérea superior, alteraciones/patología de la conciencia y pacientes que 
requirieran otra técnica que no fuera laringoscopia directa.

Previamente a la inducción anestésica, se evaluó el riesgo de dificultad para intubación traqueal (IMC, Mallampati, apertura de boca y distancia tiromentoniana). Los procedimientos de inducción e intubación se realizaron bajo los estándares de las guías francesas.

Tras la preoxigenación se llevó a cabo la ISR utilizando un hipnótico de rápida actividad (propofol, etomitado, tiopental o ketamina) y succinilcolina ( $1 \mathrm{mg} / \mathrm{kg})$. La laringoscopia se realizó en posición de hiperextensión con pala metálica de MacIntosh. Se midió el tiempo de intubación (comprendido desde la inserción del laringoscopio hasta hinchar el balón del tubo endotraqueal). La correcta colocación se examinaba con la monitorización EtCO2. Quedó a decisión del anestesiólogo: retirar la sonda nasogástrica (SNG), administración previa de un antiácido, elección del hipnótico y el uso de opioides.

- Intervención: los sanitarios tuvieron que ser entrenados previamente para aplicar una técnica correcta: presión equivalente a $30 \mathrm{~N}$ con los tres primeros dedos sobre el cartílago cricoides (4). Para asegurar un correcto doble ciego, se interpuso una sábana opaca para que el investigador interviniente no supiera si estaba en el grupo con presión o sin presión cricoidea.

- Objetivo principal: justificar que la no realización del Sellick no aumenta la aspiración pulmonar visualizada por laringoscopia directa o aspiración justo después de la intubación orotraqueal en ISR.
Modelo estadístico: Debido al amplio rango de aspiración pulmonar, los autores decidieron emplear los resultados publicados por Martin et al. que obtuvo una incidencia del $2.8 \%$ (5). Se estableció que la hipótesis de no inferioridad entre ambos métodos sería aceptada si el límite superior del intervalo de confianza del riesgo relativo no fuera mayor a $50 \%$ (RR 1.5$)$. Una diferencia de menos del $50 \%$ se consideró clínicamente insignificante porque la aspiración es un evento raro que puede ocurrir a pesar del uso de la presión cricoidea y también porque la presión en sí misma está asociada con efectos adversos.

El tamaño de la muestra se calculó en 1717 pacientes por brazo de estudio, para un total de 3434. Finalmente se estableció el objetivo de alcanzar un tamaño muestral 3500 , teniendo en cuenta que aproximadamente un 1.5\% de pacientes abandonarían el estudio. Las variables cualitativas fueron analizadas usando Pearson $\mathrm{X}^{2}$, test exacto de Fisher o Cochrane-Armitage; $\mathrm{y}$, las variables continuas fueron comparadas con el test de Wilcoxon.

\section{Resultados}

Participaron 10 centros reclutando un total de 3472 pacientes, los cuales fueron aleatorizados $1: 1 . \quad \mathrm{La}$ distribución final fue de 1735 personas en el grupo con Sellick y 1736 en el grupo sin intervención.

El estudio no consiguió demostrar el objetivo principal: la no inferioridad entre realizar la ISR con o sin presión cricoidea. En el grupo en el que se le realizó el Sellick ocurrió en 10 pacientes $(0,6 \%)$ mientras que en el grupo que no se realizó ocurrió en 9 pacientes $(0,5 \%)$ con RR 0.90 y IC $95 \%$ $0.33-2.38 \%$. Por tanto, la no inferioridad no pudo ser demostrada debido a que el límite alto del intervalo 
de confianza excedía el límite fijado de no inferioridad de $1.5 \%$.

En cuanto a los planteamientos secundarios estudiados de posibilidad de neumonía en $24 \mathrm{~h}$, neumonía severa, estancia hospitalaria y mortalidad no se demostraron cambios significativos en los dos grupos. Aun así, la incidencia de intubación traqueal dificultosa fue mayor en el grupo con presión cricoidea, aunque no se obtuvo significación estadística. En esta línea, también se observó en este grupo peor clasificación de los pacientes en la escala Cormack-Lehane. A su vez, tras solicitar la finalización de la presión en un alto porcentaje se mejoraba la visualización glótica. Por último, los tiempos de intubación fueron más largos en el grupo con presión, y hubo un mayor número de pacientes que requirieron dos intentos de intubación.

\section{Discusión}

Alrededor de la maniobra Sellick sigue habiendo múltiples controversias $\mathrm{y}$ desacuerdos. Aun así, muchas guías sobre el manejo de la vía aérea continúan recomendado dicha técnica durante la ISR. Por ejemplo, la guía británica ("Difficult Airway Society $(D A S)$ ”) recomienda su aplicación y lo establece como estándar en la ISR en el Reino Unido. Pero recoge las complicaciones asociadas y afirma la posibilidad de aumento de regurgitación por disminución del tono en el esfínter esofágico inferior. Y concluye, "si los intentos iniciales de laringoscopia son difíciles durante la ISR, se debe liberar la presión" (6). En el caso de la guía desarrollada recientemente por la Sociedad Española de Anestesiología y Reanimación cabe destacar que no se hace referencia activa sobre la maniobra. Aun así, esta guía adopta como modelos la inglesa y la canadiense. Por tanto, se sigue manteniendo la indicación de realizar la presión cricoidea durante la ISR ante pacientes con alto riesgo de aspiración con un grado de recomendación fuerte, Nivel de evidencia C (7).

Pero este estudio, pese a no demostrar la no inferioridad en cuanto aspiración pulmonar, es importante por tratar de estudiar aquellas prácticas catalogadas como estándares. Es de esos estudios que ponen en jaque aquellas dinámicas o actividades rutinarias que se llevan realizando como estándares en la práctica sin ser analizados, simplemente "porque siempre se han hecho así". Incluso los propios autores en la discusión afirman: "Although the cricoid pressure has been used in clinical practice for decades and is recommended by most countries during $R S F^{\prime}$. Por ello, es un estudio atrevido que ha intentado cambiar la manera y forma de trabajar de siempre para esclarecer y extender los límites de la ciencia en una práctica que roza el porque sí.

Además, debemos tener en cuenta que el ensayo clínico ha sido diseñado como no inferioridad. Este tipo de estudio analiza la eficacia de un tratamiento experimental frente al tratamiento probado, siendo el experimental no necesariamente más efectivo. El problema de haber escogido esta metodología es que la maniobra de Sellick (utilizado como tratamiento control activo) no ha sido probado en ningún estudio con evidencia suficiente para que así sea utilizado. Pero ¿qué consideramos importante del estudio? ¿qué limitaciones tiene este ensayo clínico?

1. Es el primer estudio randomizado que clasifica a los pacientes con alto riesgo de regurgitación.

2. Continua con la tendencia de baja incidencia de aspiración pulmonar ante una inducción anestésica (tanto si haya 
o no presión cricoidea) y, afirma, que aun habiendo entrenado al personal sanitario para hacer adecuadamente la presión sigue existiendo una gran dificultad en su realización de forma correcta.

3. No podemos extrapolar las conclusiones a la población pediátrica ni obstétrica, puesto que fueron criterio de exclusión. De la misma manera, los resultados de este trabajo no deberían aplicarse a otras intubaciones de urgencia fuera del bloque quirúrgico.

4. El estudio se realizó con succinilcolina como relajante muscular para la inducción de secuencia rápida. En la actualidad muchos anestesiólogos utilizan el rocuronio a $1-1,2 \mathrm{mg} / \mathrm{kg}$ para lograr una correcta inducción obteniendo los mismos resultados.

5. Se analiza como resultado principal la aspiración pulmonar intraoperatoria, pero cada paciente tiene un periodo de seguimiento hasta el alta o exitus, lo que consideramos suficiente para evaluar la incidencia de todas las complicaciones.

6. En el estudio se deja a decisión del anestesiólogo la colocación de la SNG y el uso de opioides. Se sabe que a mayor tiempo de colocación de SNG más probabilidad de relajación del esfínter inferior, al igual que ocurre con la utilización de los opioides. Este aspecto es relevante porque no se llevaron a cabo análisis de subgrupos en función de estas variables que podrían haber tenido influencia en los resultados.

7. Algo parecido ocurre con los antiácidos, los cuales no van a hacer que el paciente no se broncoaspire, pero si disminuyen las consecuencias $y$ posibles complicaciones si se produce. Hubiera sido interesante también un análisis específico de este aspecto.

\section{Conclusión}

Es un estudio con un importante tamaño muestral y bien diseñado que no ha conseguido probar la hipótesis de no inferioridad. Se necesitan estudios similares en población obstétrica y en intubaciones de emergencia fuera del ámbito intraoperatorio.

Con este estudio no respondemos a la pregunta de si el Sellick es de verdad una maniobra que ayuda o beneficia al paciente sin causar daños. "En investigación, si queremos dilucidar el camino e ir mostrando nuevas herramientas para mejorar en nuestras actividades, tenemos que combatir los límites negativos de la ciencia" (Rescher, 1984).

\section{Bibliografía}

1. Jefe EE. ¿Todavía podemos realizar la compresión en el cartílago cricoides? 2012;62:2012. (HTML)

2. Boyd CR, Schroeder C, Tolson MA. Initial management of pediatric trauma. Aeromed J. 1989;4(1):16-26. (HTML)

3. Durieux. Downloaded from anesthesiology.pubs.asahq.org by guest on 01/28/2019. Anesthesiology. 1995;31(4):305-9.

4. Birenbaum A, Hajage D, Roche S, Ntouba A, Eurin M, Cuvillon P, et al. Effect of Cricoid Pressure Compared with a Sham Procedure in the Rapid Sequence Induction of Anesthesia: The IRIS Randomized Clinical Trial. JAMA Surg. 2019;154(1):9-17. (PubMed)

5. Martin LD, Mhyre JM, Shanks AM, Tremper KK, Kheterpal S. 3,423 emergency tracheal intubations at a university hospital: Airway outcomes and complications.

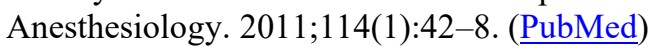

6. Cartagena NRGU de. Revista Cubana de Medicina Intensiva y Emergencias. Rev Cuba Med Intensiva y Emergencias [Internet]. 2018;17(0):57-62. Available from: http://www.revmie.sld.cu/index.php/mie/a rticle/view/551/html_172 
7. Ovassapian, A.; Salem M. Sellick Maneuver: To Do or Not To Do. Anesth Analg Gen Anesth Obstet Anesth Dig. 2011;31(1):5860. ( PubMed)

Correspondencia al autor

Carlos González Echevarría carlosgechevarria@gmail.com

Residente de $4^{\circ}$ año Anestesia y Reanimación. Hospital Universitario Severo Ochoa. Leganés, Madrid.
María Jesús Domínguez Bronchal autor@anestesiar.org

FEA Servicio Anestesiología, Reanimación y Terapéutica del dolor. Complejo Hospitalario Universitario de Toledo

Aceptado para el blog en febrero de 2020 